\title{
Article \\ Controlling Stochastic Sensitivity by Feedback Regulators in Nonlinear Dynamical Systems with Incomplete Information
}

\author{
Irina Bashkirtseva (D) \\ Institute of Natural Sciences and Mathematics, Ural Federal University, 620000 Ekaterinburg, Russia; \\ Irina.Bashkirtseva@urfu.ru
}

check for

updates

Citation: Bashkirtseva, I. Controlling Stochastic Sensitivity by Feedback Regulators in Nonlinear Dynamical Systems with Incomplete Information. Mathematics 2021, 9, 3229. https:// doi.org/10.3390/math9243229

Academic Editor: Jen-Chih Yao

Received: 13 November 2021 Accepted: 11 December 2021 Published: 14 December 2021

Publisher's Note: MDPI stays neutral with regard to jurisdictional claims in published maps and institutional affiliations.

Copyright: (C) 2021 by the author. Licensee MDPI, Basel, Switzerland. This article is an open access article distributed under the terms and conditions of the Creative Commons Attribution (CC BY) license (https:// creativecommons.org/licenses/by/ $4.0 /)$.

\begin{abstract}
The problem of synthesis of stochastic sensitivity for equilibrium modes in nonlinear randomly forced dynamical systems with incomplete information is considered. We construct a feedback regulator that uses noisy data on some system state coordinates. For parameters of the regulator providing assigned stochastic sensitivity, a quadratic matrix equation is derived. Attainability of the assigned stochastic sensitivity is reduced to the solvability of this equation. We suggest a constructive algorithm for solving this quadratic matrix equation. These general theoretical results are used to solve the problem of stabilizing equilibrium modes of nonlinear stochastic oscillators under conditions of incomplete information. Details of our approach are illustrated on the example of a van der Pol oscillator.
\end{abstract}

Keywords: control; feedback regulator; random disturbances; incomplete information; stochastic sensitivity; nonlinear oscillators

\section{Introduction}

At present, the theory of control of dynamical systems is a fairly well-developed scientific field with important engineering applications [1-3]. Mathematical problems arising in this scientific direction are associated with various aspects of the analysis of dynamical systems and optimization [4,5]. Taking into account the influence of inevitably present random perturbations complicates the formulation of control problems and leads the development of adequate methods of the stochastic analysis and synthesis. A method of stochastic Lyapunov functions for the stabilization problems was developed in [6]. The theory of random differential equations in control problems is presented in [7]. Seminal works [8,9] were devoted to the optimal stochastic control. A systematic review of theory of stochastic processes in the context of the feedback control was given in [10]. Methods of the control engineering design on the base of stochastic analysis was considered in [11]. Here, recent studies can be also mentioned [12-16].

Many mathematical models of control systems contain strong nonlinearities. It is well known that in nonlinear systems, even small random perturbations can dramatically change the deterministic dynamics and give rise to unexpected stochastic phenomena such as noise-induced transitions [17-19], stochastic resonance [20,21], noise-induced chaos [22,23], and stochastic excitability [24,25].

Even the analysis of nonlinear stochastic systems is a complex mathematical problem associated with solving the Fokker-Planck-Kolmogorov equation [26]. Here, various kinds of approximations are a useful tool [27]. The constructive technique of stochastic sensitivity functions, which makes it possible to approximate distribution densities near attractors at low noise [28,29], has been successfully used in the analysis of various kinds of stochastic phenomena [30-33].

In solving problems of synthesis for stochastic nonlinear systems with both continuous and discrete time, the use of the stochastic sensitivity method is also a fruitful direction. It was shown that the appearance of unacceptable large-amplitude oscillations induced by 
noise can be explained by the high sensitivity of initial deterministic attractors to random disturbances. In [34], it was shown how one can suppress such oscillations by the synthesis of the appropriate regulator that decreases the stochastic sensitivity. This approach has been developed through consistent studies [35-38]. Note that in the case of complete information, the construction of such a regulator was reduced to the solution of linear matrix equations.

However, in practice, the complete information on the current system state usually is not available: only some coordinates can be measured and, moreover, this data contains random errors [39]. Therefore, to develop a theory of the stochastic sensitivity synthesis for nonlinear stochastic systems with incomplete information is of particular interest [40-42].

The present paper aims to study the problem of the the stochastic sensitivity synthesis in control dynamical systems with random disturbances. We will focus on a class of static feedback regulators that use noisy data on some system state coordinates.

In Section 2, we present results of the asymptotic analysis of deviations of random solutions from the equilibrium in the general nonlinear control system. We derive a quadratic matrix equation that connects the assigned stochastic sensitivity matrix of the equilibrium and the feedback matrix of the regulator. Here, an important aspect of attainability of the assigned stochastic sensitivity is reduced to the mathematical problem of solvability of the corresponding matrix equation. Mathematical results are summarized in Theorem 1 , where explicit formulas for feedback regulators synthesizing the required stochastic sensitivity are presented. These general theoretical results are applied in Section 3 to a class of nonlinear oscillators. The efficiency of the suggested approach and the elaborated mathematical technique is illustrated by the stabilization of a stochastically forced van der Pol oscillator.

\section{Controlling Stochastic Sensitivity by a Regulator with Noisy Measurements}

Consider a general stochastic system with control in the form:

$$
\dot{x}=f(x)+g(x) u+\varepsilon \sigma(x) \xi(t) .
$$

Here, $x$ is an $n$-dimensional state, $u$ is an $l$-dimensional control input, $f(x)$ is an $n$ vector-function, $g(x)$ is an $n \times l$-matrix-function, $\xi(t)$ is an $q$-dimensional white Gaussian noise with parameters $\mathrm{E} \xi(t)=0, \mathrm{E} \xi(t) \xi^{\top}(\tau)=\delta(t-\tau) \Xi, \Xi$ is a symmetric non-negative definite $q \times q$-matrix, and $\varepsilon$ is a scalar parameter of the noise intensity. The $n \times q$-matrixfunction $\sigma(x)$ characterizes a dependence of random forcing on the system state $x$.

Let the unforced and uncontrolled system (1) (with $\varepsilon=0, u=0$ ) have an equilibrium $\bar{x}$. The stability of $\bar{x}$ is not supposed.

Let available information on the current state $x(t)$ of the system (1) be incomplete. Here, we consider a case that only the measurement $m$-vector $y(t)$ connected with the state $x(t)$ is known:

$$
y(t)=h(x(t))+\varepsilon \varphi(x) \theta(t),
$$

where $h(x)$ is $m$-vector function, $\varphi(x)$ is $m \times p$-matrix-function, $\theta(t)$ is white Gaussian $p$-vector noise, uncorrelated with $\xi(t)$, satisfying $\mathrm{E} \theta(t)=0, \mathrm{E} \theta(t) \theta^{\top}(\tau)=\delta(t-\tau) \Theta$. Here, $\Theta$ is a symmetric non-negative definite $p \times p$-matrix.

At present, regulators of very different structures are used to control systems with incomplete information. In this paper, in the problem of stabilization of the equilibrium $\bar{x}$, we focus on the case of the static regulator in the following feedback form:

$$
u=K(y-\bar{y}), \quad \bar{y}=h(\bar{x}) .
$$

Here, $K$ is a constant $l \times m$-matrix.

Let us denote by $\mathbb{K}$ a set of matrices $K$ that provide an exponential stability of the equilibrium $\bar{x}$ in the corresponding closed-loop deterministic system

$$
\dot{x}=f(x)+g(x) K(h(x)-h(\bar{x}))
$$


in some neighbourhood of the equilibrium $\bar{x}$.

Consider deviations $v(t)=x(t)-\bar{x}$ of the system (4) states $x(t)$ from the equilibrium $\bar{x}$. For these deviations, the following first approximation system can be written:

$$
\dot{v}=(F+B K C) v, \quad F=\frac{\partial f}{\partial x}(\bar{x}), \quad B=g(\bar{x}), \quad C=\frac{\partial h}{\partial x}(\bar{x}) .
$$

As follows from (5), the set $\mathbb{K}$ is determined as

$$
\mathbb{K}=\left\{K \mid \Re\left(\lambda_{i}(F+B K C)\right)<0\right\} .
$$

Here, $\lambda_{i}(F+B K C)$ are the eigenvalues of the matrix $F+B K C$. We suppose that the set $\mathbb{K}$ is not empty. Details of the algebraic analysis of this set can be found in [43].

Let us consider now the corresponding closed-loop stochastic system. As follows from (1)-(3), this closed-loop stochastic system is written in the form:

$$
\dot{x}=f(x)+g(x) K(h(x)-h(\bar{x}))+\varepsilon(g(x) K \varphi(x) \theta(t)+\sigma(x) \xi(t)) .
$$

Let $x^{\varepsilon}(t)$ be a solution of the stochastic system (6). For the asymptotics

$$
z(t)=\lim _{\varepsilon \rightarrow 0} \frac{x^{\varepsilon}(t)-\bar{x}}{\varepsilon}
$$

of the deviation of the solution $x^{\varepsilon}(t)$ of the controlled closed-loop system (6) from the equilibrium $\bar{x}$, one can write the following system

$$
\dot{z}=(F+B K C) z+B K R \theta(t)+G \xi(t), \quad R=\varphi(\bar{x}), \quad G=\sigma(\bar{x}) .
$$

In the following mean-square analysis of the sensitivity of the equilibrium $\bar{x}$ to the random disturbances, a key role is played by the covariance matrix $V(t)=\operatorname{cov}(z(t), z(t))$. Due to uncorrelatedness of noises $\xi(t)$ and $\theta(t)$, for $V(t)$ one can write the matrix equation:

$$
\dot{V}=(F+B K C) V+V(F+B K C)^{\top}+B K \Phi K^{\top} B^{\top}+S, \Phi=R \Theta R^{\top}, \quad S=G \Xi G^{\top} .
$$

For any $K \in \mathbb{K}$, an arbitrary solution $V(t)$ of this equation has a limit:

$$
W=\lim _{t \rightarrow \infty} V(t),
$$

where $W$ is a unique stable stationary solution of the Equation (8). The matrix $W$ satisfies the following quadratic algebraic equation:

$$
(F+B K C) W+W(F+B K C)^{\top}+B K \Phi K^{\top} B^{\top}+S=0 .
$$

The matrix $W$ is called a stochastic sensitivity matrix of the equilibrium $\bar{x}$. For weak noise, this matrix gives us a first approximation of the covariance matrix of the stationary distributed solutions $\bar{x}^{\varepsilon}(t)$ of the system $(6): \operatorname{cov}\left(\bar{x}^{\varepsilon}(t), \bar{x}^{\varepsilon}(t)\right) \approx \varepsilon^{2} W$.

For any $K \in \mathbb{K}$, the regulator (3) providing an exponential stability of the equilibrium $\bar{x}$ for the deterministic system (4), forms the corresponding stochastic sensitivity matrix $W_{K}$ of this equilibrium in the closed-loop stochastic system (6).

Varying $K \in \mathbb{K}$, we can change the values of the stochastic sensitivity matrix $W_{K}$ and thereby control the variance of the states $x^{\varepsilon}(t)$ of the stochastic system (1) near the equilibrium $\bar{x}$. So, the control problem for the dispersion of random states around the equilibrium $\bar{x}$ can be reduced to the synthesis of the assigned stochastic sensitivity matrix $W$ by the appropriate regulator (3).

Denote by $\mathbb{M}$ a set of symmetric and positive definite $n \times n$-matrices. Note that not any matrix $W \in \mathbb{M}$ can be synthesized. Indeed, multiplying the Equation (9) by the projective 
matrix $P$ from the left and righ, we get for the matrix $W$ the following necessary condition of the attainability:

$$
P\left(F W+W F^{\top}+S\right) P=0 .
$$

Here, $P=I-B B^{+}$is the projective matrix, and the sign " + " means pseudoinversion. Emphasize that the condition (10) does not depend on the measurement parameters.

Let us now formulate the corresponding problem of the stochastic synthesis in terms of solving the algebraic Equation (9).

Problem of the stochastic sensitivity synthesis: For the assigned matrix $W \in \mathbb{M}$, it is necessary to find a matrix $K \in \mathbb{K}$ guaranteeing the equality $W_{K}=W$, where $W_{K}$ is a solution of Equation (9).

In many cases, not any matrix $W \in \mathbb{M}$ is attainable.

Definition 1. The element $W \in \mathbb{M}$ is said to be attainable if there exists $K \in \mathbb{K}$ that the equality $W_{K}=W$ holds.

Definition 2. The set of all attainable elements,

$$
\mathbb{W}=\left\{W \in \mathbb{M} \mid \exists K \in \mathbb{K} \quad W_{K}=W\right\}
$$

is called the attainability set for system (6).

The notion of attainability in context of stochastic sensitivity synthesis was introduced and discussed in [34].

Thus, as it follows from (9), to synthesize the assigned stochastic sensitivity matrix $W$, it is necessary to find the feedback gain $K$ satisfying the quadratic matrix equation

$$
B K \Phi K^{\top} B^{\top}+B K C W+W C^{\top} K^{\top} B^{\top}+F W+W F^{\top}+S=0 .
$$

It what follows we suppose that noise in measurements is non-singular in the sense that $\operatorname{rank} \Phi(x)=m$.

Let us consider the substitution $L=B K \Phi^{\frac{1}{2}}$. For this new matrix variable $L$, the Equation (11) can be written as

$$
L L^{\top}+\left(F+L \Phi^{-\frac{1}{2}} C\right) W+W\left(F+L \Phi^{-\frac{1}{2}} C\right)^{\top}+S=0
$$

It is convenient to rewrite the Equation (12) in the form:

$$
\left(L+W C^{\top} \Phi^{-\frac{1}{2}}\right)\left(L+W C^{\top} \Phi^{-\frac{1}{2}}\right)^{\top}-W C^{\top} \Phi^{-1} C W+F W+W F^{\top}+S=0 .
$$

Let us denote

$$
N=L+W C^{\top} \Phi^{-\frac{1}{2}}, \quad Q=W C^{\top} \Phi^{-1} C W-F W-W F^{\top}-S,
$$

then the Equation (13) can be rewritten as

$$
N N^{\top}=Q
$$

The non-negative definiteness of the matrix $Q$

$$
W C^{\top} \Phi^{-1} C W-F W-W F^{\top}-S \succeq 0 .
$$

is a necessary condition of the solvability of the Equation (14). From the decomposition $Q=A A^{\top}$, where $A$ is an $n \times m$-matrix, a general solution of the quadratic Equation (14) can be written as:

$$
N=A U \text {. }
$$


Here, $U$ is an arbitrary orthogonal $m \times m$-matrix. Finally, for the feedback matrix $K$, we get the matrix equation

$$
B K=A U \Phi^{-\frac{1}{2}}-W C^{\top} \Phi^{-1} .
$$

So, the problem of the solution of the quadratic matrix Equation (11) is reduced to the solution of the linear matrix Equation (17).

If the matrix $B$ is quadratic $(n=l)$ and has a full $\operatorname{rank}(\operatorname{rank} B=n$, then the Equation (17) has a solution

$$
K=B^{-1}\left[A U \Phi^{-\frac{1}{2}}-W C^{\top} \Phi^{-1}\right] .
$$

If $\operatorname{rank} B<n$, then it is necessary to take into account one more condition for the solvability of the Equation (17):

$$
P\left[A U \Phi^{-\frac{1}{2}}-W C^{\top} \Phi^{-1}\right]=0 .
$$

Under these conditions, the feedback matrix has the form

$$
K=B^{+}\left[A U \Phi^{-\frac{1}{2}}-W C^{\top} \Phi^{-1}\right] .
$$

Results of this Section are summarized in the following Theorem.

Theorem 1. Let noise in the system (2) be non-singular.

(a) If $\operatorname{rank}(B)=n$, then the attainability set consists of the matrices $W \in \mathbb{M}$ satisfying inequality (15).

(b) For any attainable matrix $W$, the Equation (11) has a solution (18).

If $\operatorname{rank}(B)<n$ then the attainability set consists of the matrices $W \in \mathbb{M}$ satisfying relations

(15) and (19). Under these conditions, the Equation (11) has a solution (20).

This Theorem generalizes the results presented in $[34,40]$.

Examples

Example 1. For $n=m=l=q=r=1$, the Equation (11) is written as

$$
B^{2} \Phi K^{2}+2 W B K C+2 W F+S=0 .
$$

In this one-dimensional case, the attainability condition (15) is

$$
C^{2} W^{2}-2 \Phi F W-\Phi S \geq 0 \text {. }
$$

From this quadratic inequality, taking into account the non-negativeness of $W$, we get for stochastic sensitivity $W$ the attainability condition $W \geq \bar{W}$, where

$$
\bar{W}=\frac{\Phi F+\sqrt{\Phi^{2} F^{2}+C^{2} \Phi S}}{C^{2}} .
$$

To synthesize the stochastic sensitivity $W \geq \bar{W}$, one has to use the regulator with the feedback coefficient $K$ found from the formula (18) with $U= \pm 1$ :

$$
K=-\frac{W C}{B \Phi} \pm \frac{1}{B \Phi} \sqrt{C^{2} W^{2}-2 \Phi F W-\Phi S} .
$$


In (21), $\bar{W}$ is the minimum value of the stochastic sensitivity. This value is achieved by the regulator with

$$
\bar{K}=-\frac{1}{B C}\left(F+\sqrt{F^{2}+\frac{C^{2} S}{\Phi}}\right) .
$$

Let us fix $B=C=S=1$. For this case, the function $W=W(F, \Phi)$ that determines the minimum of stochastic sensitivity is plotted in Figure 1a for different values of $F$. Plots of the feedback coefficients $K=K(F, \Phi)$ of the corresponding optimal regulators providing such a minimum stochastic sensitivity are shown in Figure $1 b$.

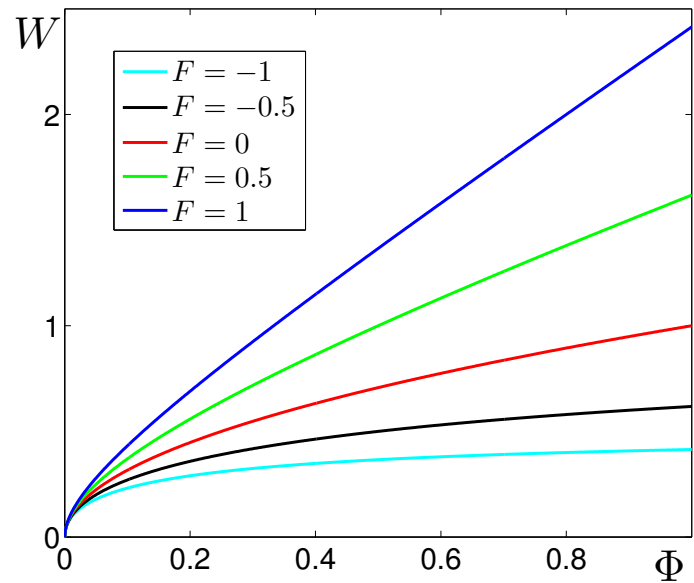

(a)

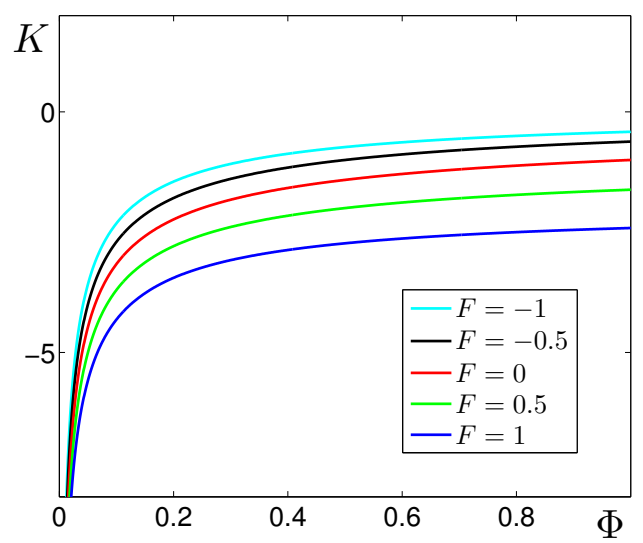

(b)

Figure 1. Plots of the functions $W=W(F, F)$ and $K=K(F, F)$ versus parameter $F$ that characterizes noise in measurement, for different $F$. Plots of the function $W=W(F, F)(\mathbf{a})$ and $K=K(F, F)(\mathbf{b})$ versus...

As can be seen, a decrease of noise intensity in measurements implies the decrease of the stochastic sensitivity.

Example 2. Consider the following cubic system

$$
\dot{x}=x-x^{3}+u+\varepsilon \xi(t), \quad \mathrm{E} \xi(t)=0, \mathrm{E} \xi(t) \xi(\tau)=\delta(t-\tau),
$$

with the measurement

$$
y=x+\varepsilon \varphi \theta(t), \quad \mathrm{E} \theta(t)=0, \mathrm{E} \theta(t) \theta(\tau)=\delta(t-\tau) .
$$

Here, $\xi(t)$ and $\theta(t)$ are uncorrelated white Gaussian noises. The deterministic uncontrolled system $(\varepsilon=0, u=0)$ possesses three equilibria: unstable $\bar{x}_{1}=0$, and stable $\bar{x}_{2,3}= \pm 1$. 
Our control aims to stabilize the equilibrium $\bar{x}_{1}=0$ using the regulator $u=K y$ that minimizes the stochastic sensitivity.

In this example, $F=B=C=S=1$ and $\Phi=\varphi^{2}$. As follows from (21) and (22), the minimum value of the stochastic sensitivity is

$$
\bar{W}=\varphi^{2}+\sqrt{\varphi^{4}+\varphi^{2}} .
$$

The feedback coefficient of the optimal regulator is written as

$$
\bar{K}=-\frac{\bar{W}}{\varphi^{2}} .
$$

Let us fix $\varepsilon=0.1, \varphi=0.1$. In the system without control, a random trajectory starting from the unstable equilibrium $\bar{x}_{1}$ transits to the vicinity of the stable equilibrium (see the red trajectory in Figure 2). In numerical simulations of random trajectories, we used a standard Euler-Maruyama scheme with the time step 0.001 .

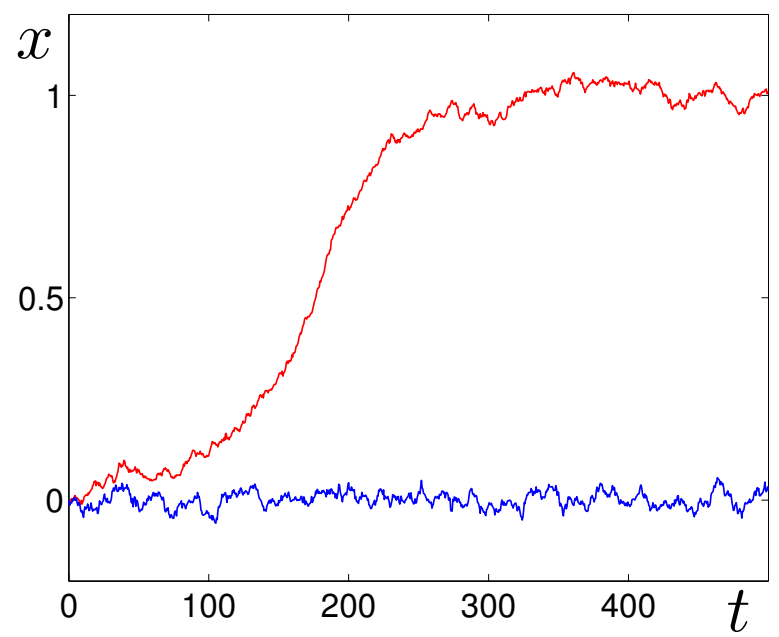

Figure 2. Cubic model with $\varepsilon=0.1, \varphi=0.1$ : random trajectory (red) of the system without control $(u=0)$ and random trajectory (blue) of the controlled system with the regulator that provides minimum stochastic sensitivity.

For $\varphi=0.1$, according to the Formula (23), the minimum value of the stochastic sensitivity is $\bar{W}=0.1105$. The corresponding optimal regulator providing this stochastic sensitivity, has the feedback coefficient $K=-11.05$ (see Formula (24)). Results of the application of this regulator are shown in Figure 2 by blue. As one can see, a sample random trajectory of the controlled system exhibits small-amplitude oscillations near $\bar{x}_{1}=0$.

\section{Synthesis of Stochastic Sensitivity in Nonlinear Oscillators}

In this Section, we consider a nonlinear oscillator with random disturbances and control input:

$$
\ddot{x}=f(x, \dot{x})+u+\varepsilon \sigma(x, \dot{x}) \xi(t) .
$$

Here, $f$ and $\sigma$ are scalar functions, $\xi(t)$ is white Gaussian noise with parameters $\mathrm{E} \xi(t)=0, \mathrm{E} \xi(t) \xi(\tau)=\delta(t-\tau)$, and $\varepsilon$ is the noise intensity.

The Equation (25) can be rewritten as a 2D-system:

$$
\begin{aligned}
& \dot{x_{1}}=x_{2}, \\
& \dot{x_{2}}=f\left(x_{1}, x_{2}\right)+u+\varepsilon \sigma\left(x_{1}, x_{2}\right) \xi(t) .
\end{aligned}
$$


Assume that the only coordinate $x_{1}$ is measured with random errors:

$$
y=x_{1}+\varepsilon \varphi\left(x_{1}\right) \theta(t),
$$

where $\theta(t)$ is white Gaussian noise with parameters $\mathrm{E} \theta(t)=0, \mathrm{E} \theta(t) \theta(\tau)=\delta(t-\tau)$. We suppose that $\xi(t)$ and $\theta(t)$ are uncorrelated.

Let $\left(\bar{x}_{1}, 0\right)$ be an equilibrium of the uncontrolled deterministic system $(26)(u=0, \varepsilon=$ 0 ). Stability of this equilibrium is not supposed. To stabilize the equilibrium $\left(\bar{x}_{1}, 0\right)$, we will use the following feedback regulator:

$$
u=K\left(y-\bar{x}_{1}\right),
$$

where $K$ is a scalar feedback coefficient.

In notations of Section 2, we have

$$
\begin{gathered}
F=\left[\begin{array}{cc}
0 & 1 \\
\alpha & \beta
\end{array}\right], \quad B=\left[\begin{array}{l}
0 \\
1
\end{array}\right], \quad C=\left[\begin{array}{ll}
1 & 0
\end{array}\right], \quad S=\left[\begin{array}{cc}
0 & 0 \\
0 & \sigma^{2}
\end{array}\right], \\
\alpha=\frac{\partial f}{\partial x_{1}}\left(\bar{x}_{1}, 0\right), \quad \beta=\frac{\partial f}{\partial x_{2}}\left(\bar{x}_{1}, 0\right), \quad \sigma=\sigma\left(\bar{x}_{1}, 0\right), \quad \varphi=\varphi\left(\bar{x}_{1}, 0\right) .
\end{gathered}
$$

Eigenvalues of the matrix

$$
F+B K C=\left[\begin{array}{cc}
0 & 1 \\
\alpha+K & \beta
\end{array}\right]
$$

satisfy the equation

$$
\lambda^{2}-\beta \lambda-(\alpha+K)=0 .
$$

We suppose that the regulator (28) stabilizes the equilibrium $\left(\bar{x}_{1}, 0\right)$ in the deterministic system (26) and (27) for $\varepsilon=0$, so it holds that

$$
\beta<0, \quad \alpha+K<0 .
$$

In what follows, we consider the case $\beta<0$. So, the set of feedback coefficients of stabilizing regulators is $\mathbb{K}=\{K \mid K<-\alpha\}$.

Now, let us turn to the problem of the stochastic sensitivity synthesis. Let $W=$ $\left[\begin{array}{cc}w_{1} & w_{2} \\ w_{2} & w_{3}\end{array}\right]$ be an assigned positive definite stochastic sensitivity matrix.

For the system (26), we have the following form of the projective matrix:

$$
P=\left[\begin{array}{ll}
1 & 0 \\
0 & 0
\end{array}\right]
$$

So, as it follows from (10), the element $w_{2}=0$, and therefore the attainable stochastic sensitivity matrix $W$ for system (26) has a be diagonal: $W=\operatorname{diag}\left[w_{1}, w_{3}\right]$.

The next step in attainability analysis is related to the matrix $Q$. In considered case,

$$
Q=\left[\begin{array}{cc}
\frac{w_{1}^{2}}{\varphi^{2}} & -\alpha w_{1}-w_{3} \\
-\alpha w_{1}-w_{3} & -2 \beta w_{3}-\sigma^{2}
\end{array}\right]
$$

The condition (15) of non-negative definiteness of the matrix $Q$ can be written as

$$
2 \beta w_{3}+\sigma^{2} \leq 0, \quad-\frac{w_{1}^{2}}{\varphi^{2}}\left(2 \beta w_{3}+\sigma^{2}\right)-\left(\alpha w_{1}+w_{3}\right)^{2} \geq 0 .
$$


Taking into account $\beta<0$, we have the restriction for the element $w_{3}$ :

$$
w_{3} \geq-\frac{\sigma^{2}}{2 \beta} .
$$

Further, for $Q$, we have to find the decomposition $Q=A A^{\top}$ where $A$ is $2 \times 1$-matrix. So, for elements $a_{1}, a_{2}$ of the matrix $A=\left[\begin{array}{l}a_{1} \\ a_{2}\end{array}\right]$, the following system can be written:

$$
\begin{aligned}
& a_{1}^{2}=\frac{w_{1}^{2}}{\varphi^{2}} \\
& a_{1} a_{2}=-\alpha w_{1}-w_{3} \\
& a_{2}^{2}=-2 \beta w_{3}-\sigma^{2} .
\end{aligned}
$$

This system of three equations for two unknown $a_{1}$ and $a_{2}$ is solvable if and only if

$$
\left(\alpha+\frac{w_{3}}{w_{1}}\right)^{2} \varphi^{2}+2 \beta w_{3}+\sigma^{2}=0
$$

and has a solution

$$
a_{1}=\frac{w_{1}}{\varphi}, \quad a_{2}=-\left(\alpha+\frac{w_{3}}{w_{1}}\right) \varphi
$$

So, the restrictions

$$
\left(\alpha+\frac{w_{3}}{w_{1}}\right)^{2} \varphi^{2}+2 \beta w_{3}+\sigma^{2}=0, \quad w_{3} \geq-\frac{\sigma^{2}}{2 \beta}
$$

give us necessary and sufficient conditions of attainability for elements $w_{1}, w_{3}$ of the stochastic sensitivity matrix $W$. Note that these conditions determine some curve in the plain $\left(w_{1}, w_{3}\right)$.

In considered case, from the general Formulas (19) and (20), it follows that

$$
K=-\alpha-\frac{w_{3}}{w_{1}} .
$$

Thus, the regulator (28) with such a coefficient $K \in \mathbb{K}$ synthesizes in the system (26) with measurements (27) a diagonal stochastic sensitivity matrix $W=\operatorname{diag}\left[w_{1}, w_{3}\right]$, where elements $w_{1}$ and $w_{3}$ are connected by the attainability conditions (29).

Example 3. Stabilization of the stochastic van der Pol oscillator.

Let us consider how general results presented above can be applied to the solution of the stabilization problem of the equilibrium $\bar{x}_{1}=\bar{x}_{2}=0$ in the randomly forced van der Pol oscillator

$$
\begin{aligned}
& \dot{x_{1}}=x_{2}, \\
& \dot{x_{2}}=-x_{1}+\beta\left(1-x_{1}^{2}\right) x_{2}+u+\varepsilon \sigma \xi(t)
\end{aligned}
$$

with measurements

$$
y=x_{1}+\varepsilon \varphi \theta(t)
$$

and feedback control $u=K y$. In this model, we restrict ourselves by additive random disturbances where $\xi(t)$ and $\theta(t)$ are uncorrelated white Gaussian noises with parameters $\mathrm{E} \xi(t)=\mathrm{E} \theta(t)=$ $0, \mathrm{E} \xi(t) \xi(\tau)=\mathrm{E} \theta(t) \theta(\tau)=\delta(t-\tau)$.

The equilibrium $(0,0)$ in the deterministic system without control $(\varepsilon=0, u=0)$ is exponentially stable for $\beta<0$. In presence of noise, the stochastic sensitivity matrix $W=\operatorname{diag}\left[w_{1}, w_{3}\right]$ of this equilibrium has equal elements $w_{1}=w_{3}=-\frac{\sigma^{2}}{2 \beta}$. For small 
negative $\beta$, the stochastic sensitivity is high. This can cause large-amplitude stochastic oscillations near the equilibrium. Such oscillations are shown by blue in Figure $3 \mathrm{~b}$ for $\beta=-0.01, \sigma=1$, and $\varepsilon=0.05$. For these parameters, $w_{1}=w_{3}=50$.

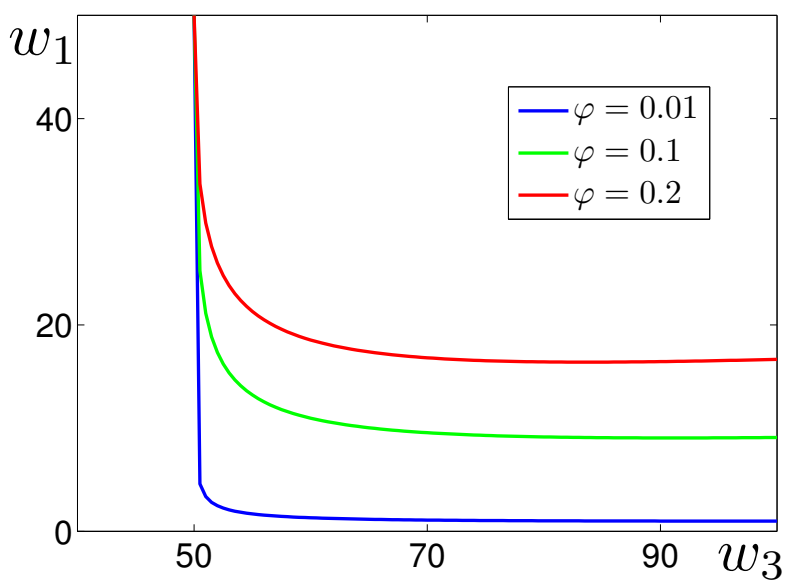

(a)

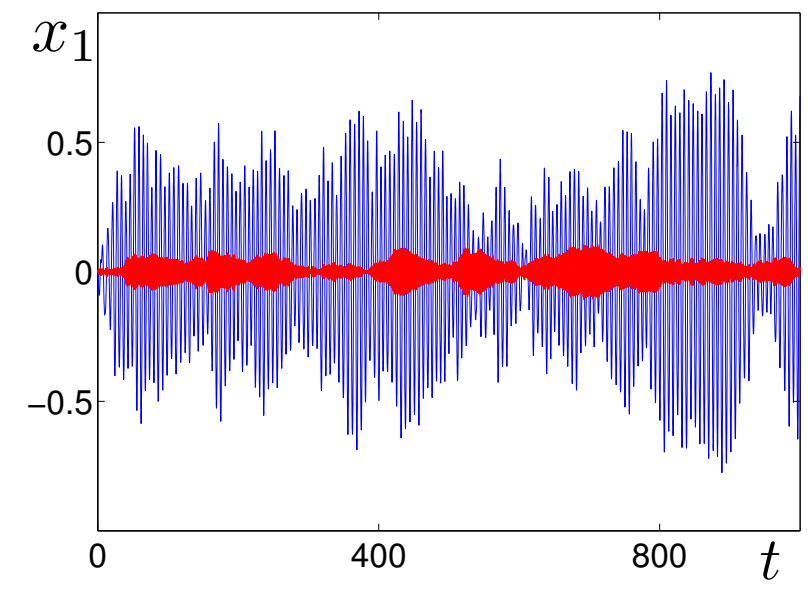

(b)

Figure 3. Stochastic van der Pol oscillator with $\beta=-0.01, \sigma=1$ : (a) plots of attainability sets for different $\varphi$; (b) random trajectory (blue) of the system without control $(u=0)$ and random trajectory (red) of the controlled system with the regulator with $K=-100$ that provides stochastic sensitivity $w_{1}=0.99, w_{3}=100$. Here, $\varepsilon=0.05, \varphi=0.01$.

Consider abilities of the regulator $u=K y$ in conditions of the measurement (31) to change parameters $w_{1}$ and $w_{3}$ of the stochastic sensitivity. For the system (30) and (31) with $\sigma=1$, the attainability conditions (29) give us

$$
\left(\frac{w_{3}}{w_{1}}-1\right)^{2} \varphi^{2}+2 \beta w_{3}+1=0, \quad w_{3} \geq-\frac{1}{2 \beta} .
$$

As can be seen, in the considered example, the attainability set is a curve in the plain $\left(w_{3}, w_{1}\right)$ determined by relations (32). For fixed $\beta=-0.01$, these curves are shown in Figure 3 a for different $\varphi$. As can be seen, our regulator allows one essentially decrease the value $w_{1}$.

For the fixed values $\beta=-0.01, \sigma=1$, and $\varphi=0.01$, the pair $w_{1}=100 / 101$ and $w_{3}=100$ is attainable. The regulator that synthesizes such sensitivity has the feedback coefficient $K=-100$. Results of direct numerical simulation of the system (30) and (31) with such a regulator are shown by red in Figure $3 \mathrm{~b}$ for $\varepsilon=0.05$. As one can see, dispersion of random solutions is essentially reduced. 


\section{Conclusions}

A problem of controlling the dispersion of random solutions for a nonlinear stochastic system with incomplete information was considered. In this paper, we constructed regulators in a feedback form that uses noisy data on some system state coordinates only. In many practical cases, even small random disturbances can cause unwanted stochastic oscillations with large amplitudes. Such excitability can be explained by high stochastic sensitivity of the system. A key point of our approach was to reduce the stochastic sensitivity by the choice of the appropriate regulator. According to this idea, the parameters of the feedback regulator should be chosen to synthesize the assigned stochastic sensitivity of the equilibrium in the controlled system. We derived the quadratic matrix equation that connects parameters of the regulator with elements of the assigned stochastic sensitivity matrix. An important issue of our analysis was the study of attainability of the assigned stochastic sensitivity. Mathematical results were summarized in Theorem 1, where explicit formulas for feedback regulators synthesizing the required stochastic sensitivity were derived using matrix analysis and the pseudoinversion technique. We successfully applied these general results to the important class of nonlinear stochastic oscillators. Details of our approach and elements of the elaborated mathematical theory were illustrated on the example of the van der Pol oscillator.

Funding: The work was supported by Russian Foundation for Basic Research (20-01-00165).

Institutional Review Board Statement: Not applicable.

Informed Consent Statement: Not applicable.

Data Availability Statement: The data presented in this study are available on request from the corresponding author.

Conflicts of Interest: The author declares no conflicts of interest.

\section{References}

1. Chen, G. Controlling Chaos and Bifurcations in Engineering Systems; CRC Press: Boca Raton, FL, USA, 1999.

2. Zabczyk, J. Mathematical Control Theory; Birkhauser: Boston, MA, USA, 2008.

3. Ogata, K. Modern Control Engineering; Prentice Hall: Hoboken, NJ, USA, 2010.

4. Craven, B. Control and Optimization; Chapman and Hall/CRC: London, UK, 1998.

5. Chinchuluun, A.; Pardalos, P.M.; Enkhbat, R.; Tseveendorj, I. Optimization and Optimal Control; Springer: New York, NY, USA, 2010.

6. Kushner, H.J. Stochastic Stability and Control; Academic Press: New York, NY, USA, 1967.

7. Wonham, W. Random Differential Equations in Control Theory; Academic Press: New York, NY, USA, 1970.

8. Astrom, K.J. Introduction to the Stochastic Control Theory; Academic Press: New York, NY, USA, 1970.

9. Fleming, W.H.; Rishel, R.W. Deterministic and Stochastic Optimal Control; Springer: New York, NY, USA, 1975.

10. Sun, J.Q. Stochastic Dynamics and Control; Elsevier: Amsterdam, The Netherlands, 2006.

11. Guo, L.; Wang, H. Stochastic Distribution Control System Design: A Convex Optimization Approach; Springer: New York, NY, USA, 2010.

12. Feng, X.W. Stochastic recursive optimal control problem of reflected stochastic differential systems. Int. J. Control 2020, 93, 2187-2198. [CrossRef]

13. Rodriguez-Gonzalez, P.T.; Rico-Ramirez, V.; Rico-Martinez, R.; Diwekar, U.M. A new approach to solving stochastic optimal control problems. Mathematics 2019, 7, 1207. [CrossRef]

14. Federico, S.; Ferrari, G.; Schuhmann, P. A singular stochastic control problem with interconnected dynamics. SIAM J. Control Optim. 2020, 58, 2821-2853. [CrossRef]

15. Munoz-Carpintero, D.; Cannon, M. Convergence of stochastic nonlinear sytems and implications for stochastic model-predictive control. IEEE Tr. Autom. Control 2021, 66, 2832-2839. [CrossRef]

16. Ito, K.; Ikeda, T.; Kashima, K. Sparse optimal stochastic control. Automatica 2021, 125, 109438. [CrossRef]

17. Horsthemke, W.; Lefever, R. Noise-Induced Transitions; Springer: Berlin, Germany, 1984.

18. Moss, F.; McClintock, P.V.E. Noise in Nonlinear Dynamical Systems; Cambridge University Press: Cambridge, UK, 1989.

19. Pisarchik, A.N.; Jaimes-Reátegui, R.; Sevilla-Escoboza, R.; Huerta-Cuellar, G.; Taki, M. Rogue waves in a multistable system. Phys. Rev. Lett. 2011, 107, 274101. [CrossRef] [PubMed]

20. Anishchenko, V.S.; Astakhov, V.V.; Neiman, A.B.; Vadivasova, T.E.; Schimansky-Geier, L. Nonlinear Dynamics of Chaotic and Stochastic Systems. Tutorial and Modern Development; Springer: Berlin/Heidelberg, Germany, 2007. 
21. McDonnell, M.D.; Stocks, N.G.; Pearce, C.E.M.; Abbott, D. Stochastic Resonance: From Suprathreshold Stochastic Resonance to Stochastic Signal Quantization; Cambridge University Press: Cambridge, UK, 2008.

22. Gao, J.B.; Hwang, S.K.; Liu, J.M. When can noise induce chaos? Phys. Rev. Lett. 1999, 82, 1132-1135. [CrossRef]

23. Bashkirtseva, I.; Ryashko, L. Stochastic bifurcations and noise-induced chaos in a dynamic prey-predator plankton system. Int. J. Bifurc. Chaos 2014, 24, 1450109. [CrossRef]

24. Lindner, B.; Garcia-Ojalvo, J.; Neiman, A.; Schimansky-Geier, L. Effects of noise in excitable systems. Phys. Rep. 2004, 392, 321-424. [CrossRef]

25. Bashkirtseva, I.; Ryashko, L. Stochastic sensitivity and method of principal directions in excitability analysis of the HodgkinHuxley model. Int. J. Bifurc. Chaos 2019, 29, 1950186. [CrossRef]

26. Risken, H. The Fokker-Planck Equation. Methods of Solution and Applications; Springer: Berlin, Germany, 1984.

27. Freidlin, M.I.; Wentzell, A.D. Random Perturbations of Dynamical Systems; Springer: Berlin, Germany, 2012.

28. Ryashko, L.; Bashkirtseva, I. Analysis of excitability for the FitzHugh-Nagumo model via a stochastic sensitivity function technique. Phys. Rev. E 2011, 83, 061109. [CrossRef]

29. Bashkirtseva, I. Stochastic sensitivity analysis: theory and numerical algorithms. IOP Conf. Ser. Mater. Sci. Eng. 2017, 192, 012024. [CrossRef]

30. Skurativskyi, S.; Skurativska, I. Dynamics of traveling waves in fluctuating nonlocal media. Commun. Nonlinear Sci. Numer. Simulat. 2017, 49, 9-16. [CrossRef]

31. Bashkirtseva, I.; Ryashko, L. Mixed-mode self-oscillations, stochastic excitability, and coherence resonance in flows of highly concentrated suspensions. Nonlin. Dyn. 2020, 102, 1837-1848. [CrossRef]

32. Ryashko, L. Noise-induced complex oscillatory dynamics in the Zeldovich-Semenov model of a continuous stirred tank reactor. Chaos 2021, 31, 013105. [CrossRef] [PubMed]

33. Alexandrov, D.V.; Bashkirtseva, I.A.; Crucifix, M.; Ryashko, L.B. Nonlinear climate dynamics: From deterministic behaviour to stochastic excitability and chaos. Phys. Rep. 2021, 902, 1-60. [CrossRef]

34. Ryashko, L.; Bashkirtseva, I. On control of stochastic sensitivity. Autom. Remote Control 2008, 69, 1171-1180. doi:10.1134/S00051179 08070084. [CrossRef]

35. Bashkirtseva, I.; Ryashko, L. Stabilization of stochastic cycles and chaos suppression for nonlinear discrete-time systems. Nonlin. Dyn. 2012, 67, 2505-2517. [CrossRef]

36. Bashkirtseva, I.; Chen, G.; Ryashko, L. Stabilizing stochastically-forced oscillation generators with hard excitement: A confidencedomain control approach. Eur. Phys. J. B 2013, 86, 437. [CrossRef]

37. Xu, C.; Yuan, S.; Zhang, T. Sensitivity analysis and feedback control of noise-induced extinction for competition chemostat model with mutualism. Phys. A 2018, 505, 891-902. [CrossRef]

38. Savaci, F.A.; Yilmaz, S. Controlling the rotor angle stability of single machine infinite bus system in the presence of Wiener and alpha-stable Levy type power fluctuations. Fluct. Noise Lett. 2020, 19, 2050036. [CrossRef]

39. Shen, B.; Wang, Z.; Shu, H. Nonlinear Stochastic Systems with Incomplete Information, Filtering and Control; Springer: Berlin, Germany, 2013.

40. Bashkirtseva, I.; Ryashko, L.; Chen, G. Controlling the equilibria of nonlinear stochastic systems based on noisy data. J. Frankl. Inst. 2017, 354, 1658-1672. [CrossRef]

41. Bashkirtseva, I. Method of stochastic sensitivity synthesis in a stabilisation problem for nonlinear discrete systems with incomplete information. Int. J. Control 2017, 90, 1652-1663. [CrossRef]

42. Bashkirtseva, I.; Ryashko, L.; Chen, G. Stochastic sensitivity synthesis in nonlinear systems with incomplete information. J. Frankl. Inst. 2020, 357, 5187-5198. [CrossRef]

43. Wonham, W. Linear Multivariable Control: A Geometric Approach; Springer: Berlin, Germany, 1979. 